\title{
Erminia Chiara CALABRESE, Militer au Hezbollah. Ethnographie d'un engagement dans la banlieue sud de Beyrouth
}

Paris-Beyrouth, Éditions Karthala/Ifpo, 2016, 277 p.

\section{Franck Mermier}

\section{(2) OpenEdition}

\section{Édition électronique}

URL : https://journals.openedition.org/assr/44654

DOI : 10.4000/assr.44654

ISSN : $1777-5825$

Éditeur

Éditions de l'EHESS

Édition imprimée

Date de publication : 1 décembre 2018

Pagination : 218-219

ISSN : 0335-5985

Référence électronique

Franck Mermier, «Erminia Chiara calABRESE, Militer au Hezbollah. Ethnographie d'un engagement dans la banlieue sud de Beyrouth », Archives de sciences sociales des religions [En ligne], 184 | octobredécembre 2018, mis en ligne le 01 décembre 2018, consulté le 16 janvier 2022. URL : http:// journals.openedition.org/assr/44654 ; DOI : https://doi.org/10.4000/assr.44654

Ce document a été généré automatiquement le 16 janvier 2022.

(c) Archives de sciences sociales des religions 


\title{
Erminia Chiara CALABRESE, Militer au Hezbollah. Ethnographie d'un engagement dans la banlieue sud de Beyrouth
}

Paris-Beyrouth, Éditions Karthala/Ifpo, 2016, 277 p.

\author{
Franck Mermier
}

\section{RÉFÉRENCE}

Erminia Chiara CALABRESE, Militer au Hezbollah. Ethnographie d'un engagement dans la banlieue sud de Beyrouth, Paris-Beyrouth, Éditions Karthala/Ifpo, 2016, 277 p.

1 L'ouvrage d'Erminia Chiara Calabrese est issu d'une thèse de doctorat réalisée sous la direction conjointe du professeur Enric Olivé Serret et du professeur Joseph Maïla au département d'histoire et d'histoire de l'art de l'Universitat Rovira I Virgili (Tarragona, 2014). Celle-ci est le résultat d'une enquête de terrain menée entre 2005 et 2011 au Liban. Une préface rédigée par Sabrina Mervin expose l'importance de cette recherche qui a dû éviter, entre autres, l'écueil des stéréotypes entretenus sur le Hezbollah. Si seuls les négatifs sont mentionnés, on peut cependant avancer que ceux qui relèvent d'une appréciation positive sont tout autant biaisés. Pourtant, la bibliographie sur ce parti est relativement abondante si on la compare avec la liste des travaux ayant traité des autres forces politiques au Liban. Il est vrai que son rôle central dans le jeu politique libanais et régional et la fascination que sa force militaire et son organisation exercent ont suscité de nombreux écrits.

Entre les ouvrages à charge et à décharge reflétant des positionnements politiques opposés, sur le conflit israélo-palestinien ou sur la situation politique libanaise, plusieurs travaux de sciences sociales se sont efforcés d'analyser, selon une combinaison variable de distance et d'empathie, l'organisation du parti et l'univers 
symbolique et culturel de la "résistance islamique». Parmi ces derniers, l'ouvrage d'Erminia Chiara Calabrese mérite une attention particulière du fait qu'il restitue, de manière remarquable, les motivations et les conséquences du militantisme et de l'appartenance à une "société partisane ». De fait, ce livre, qui porte donc sur les modalités de l'engagement politique au sein du Hezbollah dans la banlieue sud de Beyrouth, explore ce thème à travers les récits tirés d'une centaine d'entretiens conduits avec des militants et des partisans. Un travail patient et rigoureux d'élaboration théorique, à partir d'une approche inspirée par les courants les plus récents de la sociologie de l'engagement, permet de restituer au plus près les paroles et les trajectoires de vie de ces derniers sans que, pour autant, le livre se réduise à un recueil de témoignages.

3 L'auteure a eu d'autant plus de mérite de recueillir ce matériau inédit que les conditions d'exercice d'une enquête dans cette zone après la guerre de 2006 étaient très difficiles, et marquées par la méfiance compréhensible des militants. Les modalités de l'engagement sont étudiées en relation avec un contexte historique dominé par les guerres du Liban et par l'occupation israélienne du sud du pays. L'analyse des pratiques militantes est en outre reliée à une grammaire politique et symbolique inscrite dans un territoire urbain, la banlieue sud de Beyrouth, considérée par le Hezbollah comme la capitale de la Résistance. L'approche compréhensive choisie par Erminia Chiara Calabrese permet d'apporter une vision originale de cette société partisane. Elle met ainsi en exergue la pluralité des motivations, des parcours de vie et des types d'engagement, tout en reconstituant le système quasi-liturgique qui conditionne et entretient la mobilisation politique. De fait, on ne peut dissocier la lutte du Hezbollah contre Israël et sa défense du régime assadien en Syrie de son rôle de représentant de la communauté chiite qu'il partage avec son rival et allié le mouvement Amal. On peut aussi le percevoir comme un instrument de revanche sociale et culturelle des chiites libanais dont l'efficacité s'appuie aussi sur son inféodation à la République islamique d'Iran (reconnaissance du Guide suprême iranien).

4 Sans surprise, le premier chapitre revient sur le contexte historique et retrace, jusqu'à la guerre de 2006, l'histoire de l'implantation du Hezbollah au sein de la communauté chiite et du système confessionnel libanais. Les chapitres 2 et 3 explorent les modalités et les dispositifs de socialisation et d'engagement au sein du parti en relation avec les contextes historique (de l'occupation et de la guerre) et sociologique (banlieue sud, institutions éducatives et professionnelles). L'adoption d'une perspective genrée permet de plonger dans l'univers des militantes et partisanes et de saisir comment l'engagement féminin diffère et se rapproche de celui des hommes. Le chapitre 4 est consacré à la "grammaire symbolique du Hezbollah et de ses pratiques religieuses " dans la banlieue sud, le terrain favori des célébrations et cérémonies propres au Hezbollah. La question de l'esthétisation du politique dont il est aussi question dans ce chapitre concerne les symboles islamiques et chiites qui sont convoqués et suscitent sentiment d'appartenance et attachement émotionnel. La figure de Hassan Nasrallah, le dirigeant du parti, fait l'objet du cinquième chapitre qui traite de son parcours militant et de sa dimension charismatique comme facteur de cohésion interne. Le sixième et dernier chapitre analyse les constituants d'une « culture de la résistance » qui implique un « mode de vie ». On pourrait ajouter un « style de vie » qui marque la banlieue sud à travers son iconographie, son ambiance urbaine, ses lieux de sociabilité et qui en font à la fois un territoire relié à l'ensemble de l'agglomération beyrouthine et un lieu à part fortement identifié au Hezbollah et à son appareil sécuritaire. Une annexe bienvenue 
est la traduction en français d'un livre pour enfants sur la vie de Hassan Nasrallah. Le cahier photographique placé au milieu de l'ouvrage est consacré à l'iconographie politique du Hezbollah et aux destructions de la guerre de 2006 dans cette même zone du grand Beyrouth.

5 Une composition harmonieuse et une écriture fluide et sans jargon rendent la lecture de ce livre agréable. L'auteure a su construire un récit clair et cohérent à partir d'un vaste matériau ethnographique qui parvient à lier les formes de subjectivation politique et celles afférentes aux dispositifs organisationnels qui entretiennent et perpétuent la société partisane du Hezbollah. L'ouvrage réussit magistralement à nous faire pénétrer dans cet entre-soi partisan qui ne peut résumer la banlieue sud mais qui la marque profondément. Un des objectifs de l'ouvrage était «de déconstruire l'imaginaire politique qui présente les militants de ce parti comme des individus pauvres, très religieux, des "terroristes" ».

6 Maintenant que tout cela est " déconstruit » et que le vécu des militants a été restitué dans une part de sa complexité, ne faudrait-il pas s'appliquer à construire un autre puzzle pas seulement narratif sur les contradictions entre la réalité et l'idéal ? On ne trouvera ainsi pas de voix dissidentes dans ce livre, qu'elles soient celles de déçus du Hezbollah ou d'opposants à ce dernier. Quelques ouvrages libanais, peu nombreux, montrent aussi la face cachée du Hezbollah, ce qui mériterait certainement une étude future. Ce n'était certes pas le propos de ce livre centré sur les voies par lesquelles le Hezbollah fait société, pour utiliser une expression en vogue. Il reste que cet ouvrage constitue une contribution très précieuse non seulement à l'étude du Hezbollah mais aussi aux modes d'actualisation de l'engagement politique dans la société libanaise et au-delà. 